\title{
Prevalence of angle class III malocclusion: A systematic review and meta-analysis
}

\author{
Daniel K. Hardy, Yltze P. Cubas, Maria F. Orellana* \\ Department of Orofacial Sciences, Division of Orthodontics, School of Dentistry, University of California, San Francisco, USA \\ Email: * OrellanaM@dentistry.ucsf.edu
}

Received 10 August 2012; revised 20 September 2012; accepted 12 December 2012

\begin{abstract}
Objective: This study seeks to review current and relevant literature on global Angle class III malocclusion prevalence. Materials and Methods: The electronic databases PubMed, ISI Web of Knowledge, and the Cochrane Database of Systematic Review were searched using specific inclusion criteria to obtain applicable articles. All pertinent references were also examined for acceptability. Results: A total of 20 articles were identified using the inclusion criteria. The prevalence of Angle class III malocclusion ranged from $0 \%$ to $26.7 \%$ in different populations reported in the literature examined. Meta-regression analysis showed no statistically significant association between prevalence rates and the method of assessment, age group and year of the study. However, much of the study-to-study variation (approximately $40 \%$ ) could be explained by population. Conclusion: These results suggest that the prevalence of Angle class III malocclusion varies greatly within different races and geographic regions. Chinese and Malaysian populations have a higher prevalence of Angle class III malocclusion compared to other racial groups, while Indian populations have a lower prevalence than all other racial groups examined.
\end{abstract}

Keywords: Malocclusion; Class III; Prevalence; Systematic Review; Meta-Analysis

\section{INTRODUCTION}

The Angle classification has prevailed over the last century as a simple, quantifiable method to test malocclusion prevalence within populations. However, what Angle defined as a "normal occlusion" should, in fact, be considered the "ideal" occlusion given the strict criteria he used in his classification. This ideal occlusion is rather uncommon and has driven researchers to disagree

\footnotetext{
"Corresponding author.
}

on how much deviation from the ideal should be accepted as normal [1].

According to the Angle classification, class III malocclusion is defined as the lower molar mesially positioned relative to the upper molar with no specifications in regards to the line of occlusion [1,2]. Nevertheless, as with all Angle's classification of malocclusion, class III malocclusion comprises several skeletal and dental components that may differ from the concept of normality. For example, it can be characterized by presenting a mandibular skeletal protrusion (mandibular prognathism), a maxillary skeletal retrusion, a combination of both, or no anteroposterior skeletal imbalances [3].

Multiple studies have documented the prevalence of Angle class III malocclusion. However, there seems to be a wide range of prevalence rates reported, usually attributed to variation among samples [4-38]. For example, several studies have indicated that Asian races have a higher prevalence of Angle class III malocclusion than other races [4-10]. In some instances population data have shown conflicting reports, such as differing studies among Nigerian children reporting Angle class III malocclusion prevalence ranging from $1.2 \%$ (1993) up to $11.8 \%$ (2004) [11-13].

In spite of the continually increasing data on malocclusion prevalence, little has been done to consolidate this information in a comprehensive and critical way [14]. Further; to the best of our knowledge, there has not been a systematic analysis of the prevalence of Angle class III malocclusion among different populations. Meta-analysis is a systematic method that uses statistical techniques for combining results from different studies to obtain a quantitative estimate of the overall effect of a particular intervention or variable on a defined outcome; metaanalysis produces a stronger conclusion than can be provided by any individual study. Although no protocol has been enacted governing the methods of Angle class III malocclusion prevalence studies, a review and metaanalysis of the available literature will be helpful in establishing guidelines for future researchers. 


\section{MATERIALS AND METHODS}

\subsection{Selection Criteria}

Types of publication-Only articles from scientific journals were selected. Abstracts, editorials, letters, theses, and other types of publications were excluded. Only articles released in the last 20 years were included. Types of studies - Only observational studies were included. Case-control studies, case reports, clinical trials, experimental laboratory studies, and studies of predictors of treatment outcome were excluded. Population-Only populations with well demarcated geopolitical or racial categories were included. Studies evaluating convenience samples were excluded. Only studies involving individuals age 11 and older were included. Studies that analyzed individuals both older and younger than 11 years of age were included only if the study separated the subjects based on age for comparison. Studies that included individuals that had previously received orthodontic treatment were excluded from this study. Classification of class III malocclusion-Studies using Angle's classification of malocclusion [2] were included in this study. Studies were included that used lateral cephalogram X-ray data, intraoral exams based on canine relationship or 1st molar relationship, or extraoral exams based on canine and 1st molar relationships from study casts.

\subsection{Search Strategy}

The search terms used to retrieve relevant journal articles were as follows: [malocclusion] AND [prevalence] AND [class III]. Search was limited based on language to only English and Spanish articles. Publications were located through a computerized search of the following databases (through February 2011): PubMed, ISI Web of Knowledge, and the Cochrane Database of Systematic Reviews.

Final selection of articles was determined independently by the authors as described below. Selection criteria were fully applied at this stage and any discrepancies between the authors were resolved through discussion. Reference lists of selected articles were hand searched to find relevant articles that the database searches may have missed.

\subsection{Systematic Assessment of Articles}

Each of the selected articles were assessed according to a format that analyzed the articles in relation to patients/ problem/population, intervention/indicator, comparison/ control, and outcome (PICO), for each of which specific questions were constructed. For each article, the study population ("P") was assessed in the light of the criteria for inclusion, the demographic features of the sample, and the sample size. The study design was described in the section reserved to questions on the study intervention ("I"). The comparison measure ("C") was assessed by the presence of any comparison groups. The study outcome ("O") was evaluated on the basis of the application of objective diagnostic criteria for class III malocclusion.

\subsection{Analysis}

Descriptive statistics (number studied, number of class III cases, year of the study, age category, race/nationality of the population studied and method of assessment) were generated using Intercooled Stata Version 11 (Stata Corp LP, College Station, TX).

Meta-analysis was performed by pooling the data and using random effects logistic regression models for the outcome occlusion. Heterogeneity in prevalence was tested using likelihood ratio Chi-bar-squared test and measured using standard deviation of the prevalence across studies.

Meta-regression analysis was conducted by including additional covariates in the random effects logistic regression model to investigate the association between prevalence rates and study characteristics including year of the study, age group, population, and method of assessment.

Forest plots displaying mean prevalence (together with 95\% confidence interval) were generated for each of 23 groups listed according to author and nationality (Figure 1). Similar plots were generated for each of 5 regional categories that resulted from pooling the 23 groups according to subcontinent: African, East Asian, European, South Asian and Middle Eastern (Figure 2). A vertical line representing the null hypothesis is displayed on each forest plot.

\section{RESULTS}

The PubMed search returned 134 total articles, including 10 listed as review articles. ISI Web of Knowledge generated 52 total articles, 16 of which did not appear in the PubMed. The Cochrane search produced 2 articles.

Review of all articles produced 37 which were determined to be relevant to Angle class III malocclusion prevalence. A search of all references of these 37 articles produced an additional 42 articles that were also considered relevant. Applying the inclusion criteria for these 79 articles revealed 21 articles that were acceptable for the literature review. Two articles were excluded from the analysis because they reported the same population included in other articles (Figure 3).

The majority of articles analyzed data from African populations: Tanzania [13-15], Nigeria [10-12], Sudan [16], Senegal [17], and Kenya [18]. Four articles looked 


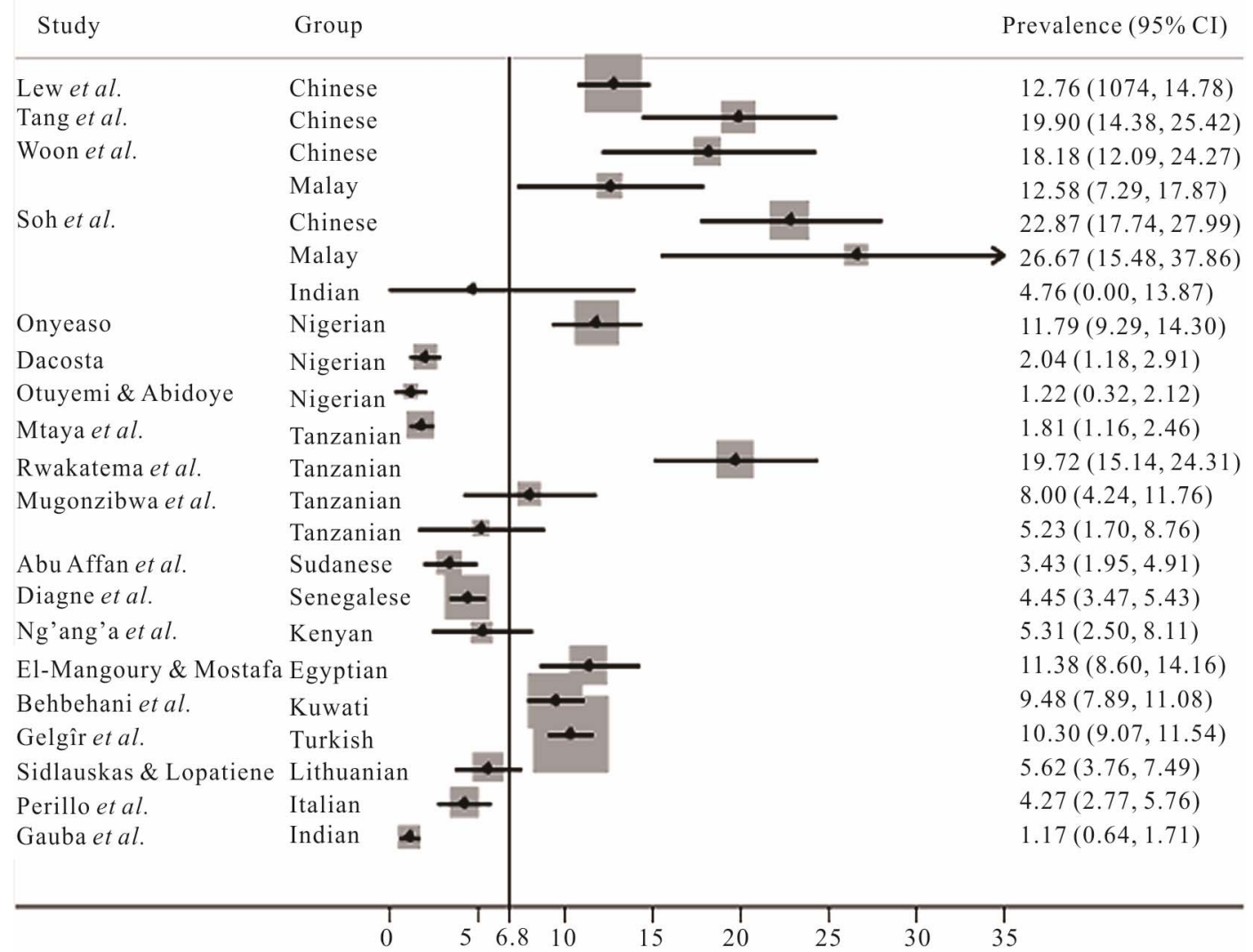

Figure 1. Forest plots of prevalence according to author and nationality.

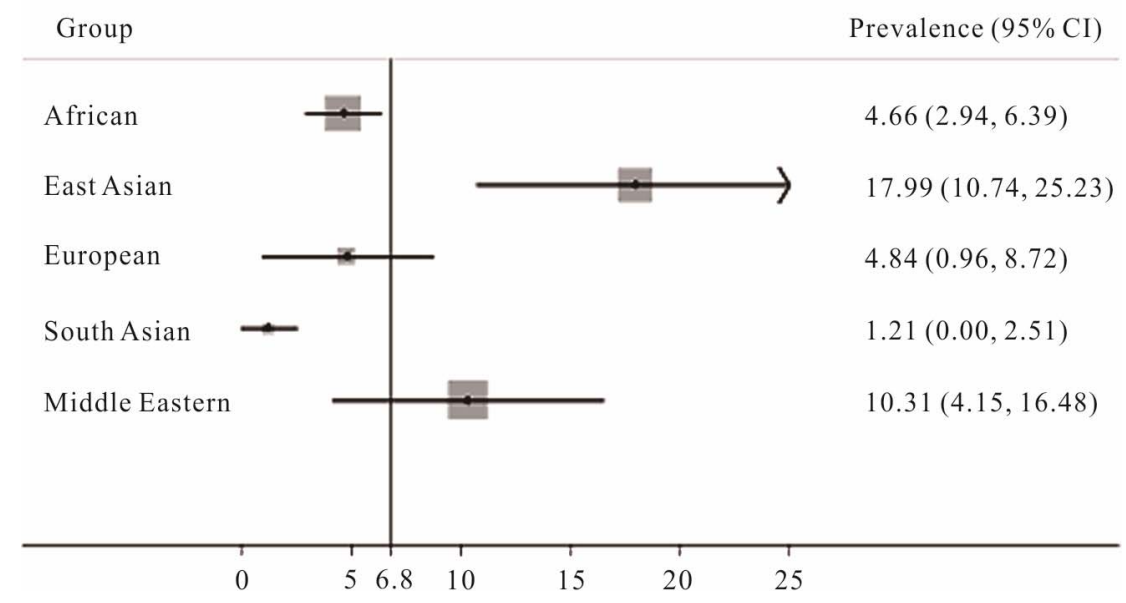

Figure 2. Forest plots of prevalence according to subcontinent.

at populations from Southeast Asia, including China [4, 5], Malaysia [7], and Singapore [8]. The articles from Singapore and Malaysia utilized a mix of Malaysian, Chinese, and Indian, which were analyzed separately in this study. Three articles reviewed populations from the Middle East: Egypt [19], Kuwait [20], and Turkey [21]. The remaining 3 articles analyzed Lithuanian [22], Italian [23], and Indian [24] populations.
The sample size of the populations ranged from 21 to 2329 and the prevalence of Angle class III malocclusion ranged from 0 to $26.67 \%$ (Table 1). The combined total sample size of all studies was 15,200 individuals, 1090 of whom exhibited Angle class III malocclusion, for an average prevalence of $7.04 \%$.

Populations from Southeast Asian countries showed the highest Angle class III malocclusion prevalence rate 


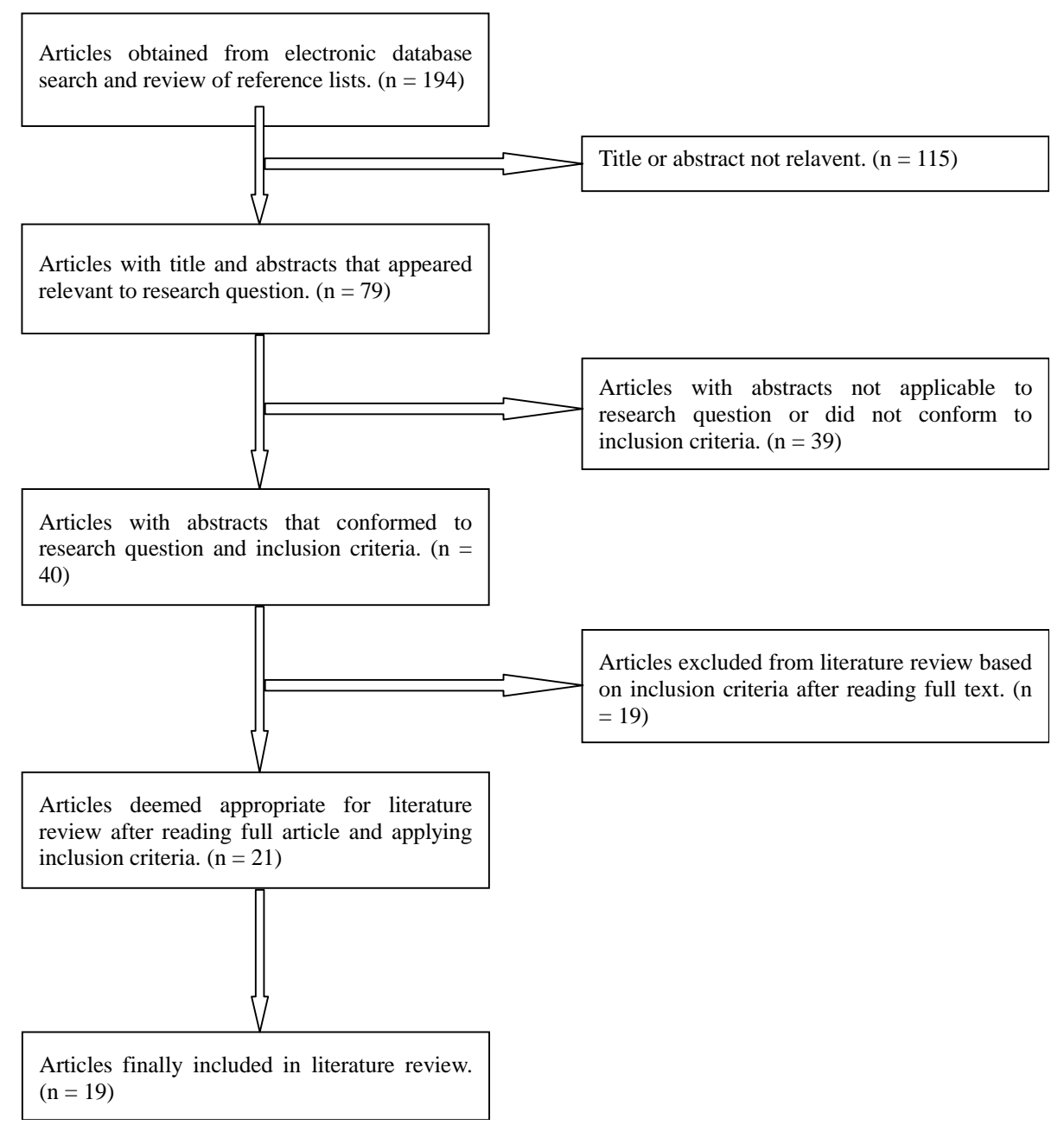

Figure 3. QUORUM flow chart of the literature search.

of $15.80 \%$, based on 1874 individuals [4-8]. Middle Eastern nations had a mean prevalence of $10.18 \%$ out of 4127 individuals [19-21]. The European studies had an average prevalence rate $4.88 \%$ out of 1290 individuals [22,23]. The African data produced a prevalence rate of $4.59 \%$ out of 7017 individuals [10-17]. Indian populations had the lowest prevalence rate of $1.19 \%$ out of 1595 individuals $[7,8,24]$.

The range of Angle class III malocclusion prevalence rates was between $1.22 \%$ and $19.72 \%$ among the African group [12,14]. Prevalence data varied most among Tanzanian populations, with prevalence rates of $1.81 \%$, $5.23 \%, 8.00 \%$, and $19.72 \%$ [13-15]. The prevalence rates were also variable among the Nigerian populations, with rates of $1.22 \%, 2.04 \%$, and $11.79 \%$ reported [10-12].

The Southeast Asian group had a range of Angle class III malocclusion prevalence between $12.58 \%$ and $26.67 \%[7,8]$. The Middle Eastern group showed a range of $9.48 \%$ and $11.38 \%[19,20]$. The Indian group had a range of $0 \%$ [7] to $4.76 \%$ [8].

Meta-regression analysis showed no statistically sig- nificant association between prevalence rates and the method of assessment, age group and year of the study. However, much of the study-to-study variation (approximately $40 \%$ ) could be explained by population when it was used as a parameter in the analysis. In fact, studyto-study variation dropped to 0.579 when population was taken into account.

Using the formula $\log$ (odds) $=\mathrm{b}_{0}+(\text { study })^{\delta}$, where $\mathrm{b}_{0}$ is the regression constant and $\delta$ is study-to-study variation expressed as an odds ratio on the logit scale (in this case, $\delta=0.979$ ), we were able calculate the increase in the odds of malocclusion for a study at 1 SD (standard deviation) above the mean. This can be expressed as $\mathrm{e}^{0.979}=2.66$, where $\mathrm{e}$ is the exponent constant. In other words, at $1 \mathrm{SD}$ above the mean, the odds of malocclusion are 2.66 times greater than at the mean (Figures $\mathbf{1}$ and 2).

\section{DISCUSSION}

Analysis of the reported prevalence of class III malocclusion requires scrutiny of the methods employed in 
Table 1. Percentage of class III malocclusions by nationalities. The 19 articles and 24 populations identified in the literature search, shown with population nationality, sample size, percent Angle class III malocclusion, method of assessment and age range for each population. Children (11 - 17 years of age), Adults (18 years of age and over).

\begin{tabular}{|c|c|c|c|c|c|c|}
\hline Author & Year & Population & $n$ & Class III n (\%) & Age Group & Method of Assessment \\
\hline Lew et al. & 1993 & Chinese & 1050 & $134(12.76)$ & Children & Clinical exam \\
\hline Tang & 1994 & Chinese & 201 & $40(19.90)$ & Adults & Models \\
\hline \multirow[t]{3}{*}{ Woon et al. } & 1989 & Chinese & 154 & $20(18.18)$ & Adults and Children & Clinical exam \\
\hline & & Malay & 151 & $19(12.58)$ & Adults and Children & Clinical exam \\
\hline & & Indian & 42 & $0(0.00)$ & Adults and Children & Clinical exam \\
\hline \multirow[t]{3}{*}{ Soh et al. } & 2005 & Chinese & 258 & $59(22.87)$ & Adults and Children & Models \\
\hline & & Malay & 60 & $16(26.67)$ & Adults and Children & Models \\
\hline & & Indian & 21 & $1(4.76)$ & Adults and Children & Models \\
\hline Gauba et al. & 1998 & Indian & 1532 & $18(1.17)$ & Children & Clinical exam \\
\hline Perillo et al. & 2010 & Italian & 703 & $30(4.27)$ & Children & Clinical exam \\
\hline El-Mangoury \& Mostafa & 1990 & Egyptian & 501 & $57(11.38)$ & Adults & Clinical exam \\
\hline Ng'ang'a et al. & 1993 & Kenyan & 245 & $13(5.31)$ & Children and Adults & Clinical exam \\
\hline Behbehani et al. & 2004 & Kuwati & 1297 & $123(9.48)$ & Children & Clinical exam \\
\hline Šidlauskas \& Lopatienė & 2009 & Lithuanian & 587 & $33(5.62)$ & Children & Clinical exam \\
\hline Onyeaso & 2004 & Nigerian & 636 & 75 (11.79) & Children & Clinical exam \\
\hline Dacosta & 1999 & Nigerian & 1028 & $21(2.04)$ & Adults and Children & Clinical exam \\
\hline Otuyemi \& Abidoye & 1993 & Nigerian & 574 & $7(1.22)$ & Children & Clinical exam \\
\hline Abu Affan et al. & 1990 & Sudanese & 583 & $20(3.43)$ & Children & Clinical exam \\
\hline Diagne et al. & 1993 & Senegalese & 1708 & $76(4.45)$ & Adults and Children & Unknown \\
\hline Mtaya et al. & 2009 & Tanzanian & 1601 & $29(1.81)$ & Children & Clinical exam \\
\hline Rwakatema et al. & 2006 & Tanzanian & 289 & $57(19.72)$ & Children & Clinical exam \\
\hline \multirow[t]{2}{*}{ Mugonzibwa et al. } & 1990 & Tanzanian & 200 & $16(8.00)$ & Children & Clinical exam \\
\hline & & Tanzanian & 153 & $8(5.23)$ & Children & Clinical exam \\
\hline Gelgör et al. & 2007 & Turkish & 2329 & $240(10.30)$ & Children & Clinical exam \\
\hline
\end{tabular}

collection of the data. Of particular interest is the subject of inclusion criteria. The quality of the inclusion criteria ultimately affects the reliability of the prevalence data.

The selection criteria for this literature search were chosen to minimize the number of confounding variables present in class III malocclusion prevalence data. Individuals under 11 years old were excluded from this study as younger children have a greater tendency for Angle class III malocclusion as compared to older children with more erupted permanent teeth. This is most likely explained by the presence of pseudo class III malocclusion, which is a habitual established crossbite of all anterior teeth, without any skeletal discrepancy, resulting from functional forward positioning of the mandible on closure [39]. Furthermore, a mesial step predisposition in the primary dentition is not a good predictor of class III malocclusion in the permanent dentition [25].

The large variation in Angle class III malocclusion prevalence rates in this study suggests a high level of variability across geographic regions and races. Chinese and Malaysian groups showed a much higher mean prevalence rate than other racial groups: $15.69 \%$ and $16.59 \%$, respectively [4-8]. This is consistent with previous reports of higher rates of Angle class III malocclusion among Asian populations [26,27].

Angle class III malocclusion rates among the three Middle Eastern groups in this study are fairly similar. However, previous studies indicated a wide range of rates from $1.3 \%$ in Israeli Arabs [28] to $15.2 \%$ in Iranians [28]. Additionally, many different rates appear for 
Iranians: $2.1 \%, 7.8 \%, 9.2 \%$, and $15.2 \%$ [29-32]. A study of Turks [33] in 2004 showed a prevalence rate of $11.5 \%$, similar to the $10.30 \%$ reported in the 2007 study [21] included in this review. However, a 1969 study of Egyptians [34] showed a rate of $4 \%$, which is not concordant with the $11.38 \%$ rate reported for the Egyptian population in this study [19]. Agreement of previously reported Angle class III prevalence data appears to be haphazard at best among Middle Eastern populations. Furthermore, major discrepancies appear to exist among populations within individual countries.

Most of the African populations showed a relatively low prevalence, although two populations each showed a much higher rate than the rest $[10,14]$. Furthermore, the range of rates among the Tanzanian populations varied considerably [13-15]. Previously studies from Kenya, Tanzania, and Nigeria also show a large level of variation in prevalence rates (between 1\% and 16.8\%) [35,36]. This suggests a large amount of local variability within and among African countries.

The lack of European populations in this study makes it difficult to draw any generalization about prevalence rates of Angle class III malocclusion. Recent studies excluded by the selection criteria show a range of $2 \%$ to 6\% among European countries [37,38]. This suggests that Europeans have a relatively lower prevalence rate than other populations.

With respect to the prevalence of class III malocclusion in the Americas, two publications were identified. The first study, reported a prevalence of class III malocclusion of 9.1\% among Mexican-American adolescents living in Los Angeles area [40]. This study was excluded as it evaluated a convenience sample. Data from the third National Health and Nutrition Examination Survey (NHANES III) showed that in the United States, only a fraction of a percentage of all adults presented class III. This type of malocclusion was more prevalent in Mexican-Americans and African-Americans compared to Whites [41]. Because this study did not distinguish between subjects that had previous orthodontic treatment, it was also excluded from the final selection.

Defining a population can be a difficult task. We found that most studies analyze a specific city or region of a country. Such a sampling may or may not represent the country as a whole. Genetic predisposition for Angle class III malocclusions may be present in certain groups, but these populations do not necessarily fall into neat geographic areas. Many countries, such as Singapore, represent a conglomeration of individuals from many different nationalities and races. Other countries, like Nigeria and China, may have numerous local ethnic groups that are divided by language, religious belief, and cultural practices, minimizing contact between groups. Although it is tempting to generalize the national status of Angle class III malocclusion in a particular country based on a few local population studies, a more representative sampling would be needed to draw more accurate conclusions.

Scoring malocclusions is generally a subjective decision based on the training and opinions of the clinician or researcher. Additionally, many researchers may be reporting different rates of malocclusion prevalence simply because they used different methods to measure the malocclusion. Ideally, a universal system for scoring Angle class III malocclusions should be employed to better standardize epidemiological studies in this area. Furthermore, researchers should be more careful in their selection criteria for their populations to eliminate any confounding variables.

It may ultimately be impossible to accurately sample every population for a general prevalence rate from both a logistical and theoretical standpoint. Populations can always be broken down into smaller groups which may or may not represent the population as a whole. However, given these limitations, it is important to remember the need for data of Angle class III malocclusion prevalence. Angle class III malocclusions can be both socially and functionally handicapping, and identifying populations that require greater attention may help clinicians and politicians in deciding how to best address helping them find treatment.

\section{CONCLUSIONS}

- Prevalence of Angle class III malocclusion varies greatly among and within populations.

- Chinese and Malaysian populations show a relatively higher prevalence of Angle class III malocclusion while Indian populations show a relatively lower prevalence, as compared to other races.

- A more standardized protocol for reporting malocclusion prevalence data would be helpful in drawing meaningful comparisons across geographic and racial groups in the future.

\section{REFERENCES}

[1] Proffit, W.R., Fields, H.W. and Sarver, D.M. (2007) Contemporary orthodontics. Mosby, St. Louis.

[2] Angle, E.H. (1899) Classification of malocclusion. Dental Cosmos, 41, 248-264.

[3] Graber, T., Vanarsdall, R. and Vig, K. (2005) Orthodontics: Current Principles and Techniques. Mosby, St. Louis.

[4] Lew, K.K., Foong, W.C. and Loh, E. (1993) Malocclussion prevalence in an ethnic Chinese population. Australian Dental Journal, 38, 442-449. doi:10.1111/j.1834-7819.1993.tb04759.x

[5] Tang, E.L. (1994) Occlusal features of Chinese adults in Hong Kong. Australian Orthodontic Journal, 13, 159- 
163.

[6] Tang, E.L. (1994) The prevalence of malocclusion amongst Hong Kong male dental students. British Journal of Orthodontics, 21, 57-63.

[7] Woon, K.C., Thong, Y.L. and Abdul Kadir, R. (1989) Permanent dentition occlusion in Chinese, Indian and Malay groups in Malaysia. Australian Orthodontic Journal, 11, 45-48.

[8] Soh, J., Sandham, A. and Chan, Y.H. (2005) Occlusal status in Asian male adults: Prevalence and ethnic variation. Angle Orthodontist, 75, 814-820.

[9] Soh, J., Sandham, A. and Chan, Y.H. (2005) Malocclusion severity in Asian men in relation to malocclusion type and orthodontic treatment need. American Journal of Orthodontics \& Dentofacial Orthopedics, 128, 648-652. doi:10.1016/j.ajodo.2005.05.045

[10] Onyeaso, C.O. (2004) Prevalence of malocclusion among adolescents in Ibadan, Nigeria. American Journal of Orthodontics \& Dentofacial Orthopedics, 126, 604-607. doi:10.1016/j.ajodo.2003.07.012

[11] Dacosta, O.O. (1999) The prevalence of malocclusion among a population of northern Nigeria school children. West African Journal of Medicine, 18, 91-96.

[12] Otuyemi, O.D. and Abidoye, R.O. (1993) Malocclusion in 12-year-old suburban and rural Nigerian children. Community Dental Health, 10, 375-380.

[13] Mtaya, M., Brudvik, P. and Astrom, A.N. (2009) Prevalence of malocclusion and its relationship with sociodemographic factors, dental caries, and oral hygiene in 12to 14-year-old Tanzanian schoolchildren. European Journal of Orthodontics, 31, 467-476. doi:10.1093/ejo/cjn125

[14] Rwakatema, D.S., Ng'ang’a, P.M. and Kemoli, A.M. (2006) Prevalence of malocclusion among 12 - 15-yearolds in Moshi, Tanzania, using Bjork's criteria. East African Medicine Journal, 83, 372-379. doi:10.4314/eamj.v83i7.9449

[15] Mugonzibwa, E.A., Mumghamba, E., Rugarabamu, P. and Kimaro, S. (1990) Occlusal and space characteristics among 12-year-old school children in Bukoba and Moshi, Tanzania. African Dental Journal, 4, 6-10.

[16] Abu Affan, A.H., Wisth, P.J. and Boe, O.E. (1990) Malocclusion in 12-year-old Sudanese Children. Odontostomatol Trop, 13, 89-93.

[17] Diagne, F., Ba, I., Ba-Diop, K., Yam, A.A. and BaTamba, A. (1993) Prevalence of malocclusion in Senegal. Community Dentistry and Oral Epidemiology, 21, 325326. doi:10.1111/j.1600-0528.1993.tb00786.x

[18] Ng'ang'a, P.M., Karongo, P.K., Chindia, M.L. and Valderhaug, J. (1993) Dental caries, malocclusion and fractured incisors in children from a pastoral community in Kenya. East African Medical Journal, 70, 175-178.

[19] El-Mangoury, N.H. and Mostafa, Y.A. (1990) Epidemiologic panorama of dental occlusion. Angle Orthodontist, 60, 207-214.

[20] Behbehani, F., Artun, J., Al-Jame, B. and Kerosuo, H. (2005) Prevalence and severity of malocclusion in adolescent Kuwaitis. Medical Principles and Practice, 14,
390-395. doi:10.1159/000088111

[21] Gelgor, I.E., Karaman, A.I. and Ercan, E. (2007) Prevalance of malocclusion among adolescents in central Anatolia. European Journal of Dentistry, 1, 125-131.

[22] Sidlauskas, A. and Lopatiene, K. (2009) The prevalence of malocclusion among 7 - 15-year-old Lithuanian children. Medicina (Kaunas), 45, 147-152.

[23] Perillo, L., Masucci, C., Ferro, F., Apicella, D. and Baccetti, T. (2010) Prevalence of orthodontic treatment need in southern Italian schoolchildren. European Journal of Orthodontics, 32, 49-53. doi:10.1093/ejo/cjp050

[24] Gauba, K., Ashima, G., Tewari, A. and Utreja, A. (1998) Prevalence of malocclusion and abnormal oral habits in North Indian rural children. Journal of Indian Society of Pedodontics and Preventive and Dentistry, 16, 26-30.

[25] Bishara, S.E., Hoppens, B.J., Jakobsen, J.R., Kohout, F.J. (1988) Changes in the molar relationship between the deciduous and permanent dentitions: A longitudinal study. American Journal of Orthodontics and Dentofacial Orthopedics, 93, 19-28. doi:10.1016/0889-5406(88)90189-8

[26] Ishii, H., Morita, S., Takeuchi, Y. and Nakamura, S. (1987) Treatment effect of combined maxillary protraction and chincap appliance in severe skeletal Class III cases. American Journal of Orthodontics and Dentofacial Orthopedics, 92, 304-312. doi:10.1016/0889-5406(87)90331-3

[27] Yang, W.S. (1990) The study on the orthodontic patients who visited department of orthodontics, Seoul National University Hospital. Taehan Chikkwa Uisa Hyophoe Chi, 28, 811-821.

[28] Steigman, S., Kawar, M. and Ziberman, Y. (1983) Prevalence and severity of malocclusion in Israeli Arab urban children 13 to 15 years of age. American Journal of Orthodontics, 84, 337-243. doi:10.1016/S0002-9416(83)90350-0

[29] Ravanmehr, H. and Rashidi-Birgani, M. (1998) A study on prevalence of dentofacial anomalies in 12 to 14 years old students in Tehran. Journal of Dentistry of Tehran University of Medical Sciences, 11, 38-45.

[30] Danaie, S.M., Asadi, Z. and Salehi, P. (2006) Distribution of malocclusion types in 7 - 9-year-old Iranian children. Eastern Mediterranean Health Journal, 12, 236-240.

[31] Borzabadi-Farahani, A., Borzabadi-Farahani, A. and Eslamipour, F. (2009) Malocclusion and occlusal traits in an urban Iranian population. An epidemiological study of 11- to 14-year-old children. European Journal of Orthodontics, 31, 477-484. doi:10.1093/ejo/cjp031

[32] Ramezanzadeh, B.A. and Hosseiny, S.H. (2005) Evaluation of prevalence of dental malocclusion in junior high school students in the city of Neishabour in year 20022003. Journal of Dentistry of Mashhad University of Medical Sciences, 29, 57-66.

[33] Sayin, M.Ö. and Türkkahraman, H. (2004) Malocclusion and crowding in an orthodontically referred Turkish population. Angle Orthodontist, 74, 635-639.

[34] Lundström, A. and Lundström, O. (1969) A dental examination of the mixed and permanent dentitions in a Nubian population. Acta Odontologica Scandinavica, 27, 
371-386. doi:10.3109/00016356909040416

[35] Hirschowitz, A.S., Rachid, S.A. and Cleaton-Jones, P.E. (1981) Dental caries, gingival health and malocclusion in 12-year-old urban Black schoolchildren from Soweto, Johannesburg. Community Dentistry and Oral Epidemiology, 9, 87-90. doi:10.1111/j.1600-0528.1981.tb01035.x

[36] Garner, L.D. and Butt, M.H. (1985) Malocclusion in black Americans and Nyeri Kenyans. An epidemiologic study. Angle Orthodontist, 55, 139-146.

[37] Burgersdijk, R., Truin, G.J., Frakenmolen, F., Kalsbeek, H., van't Hof, M. and Mulder, J. (1991) Malocclusion and orthodontic treatment need of 15 - 74-year-old Dutch adults. Community Dentistry and Oral Epidemiology, 19, 64-67. doi:10.1111/j.1600-0528.1991.tb00111.x

[38] Willems, G., De Bruyne, I., Verdonck, A., Fieuws, S. and Carels, C. (2001) Prevalence of dentofacial characteris- tics in a Belgian orthodontic population. Clinical Oral Investigations, 5, 220-226. doi:10.1007/s007840100128

[39] Kapur, A., Chawla, H.S., Utreja, A. and Goyal, A. (2008) Early class III occlusal tendency in children and its selective management. Journal of Indian Society of Pedodontics and Preventive Dentistry, 26, 107-113. doi:10.4103/0970-4388.43191

[40] Silva, R.G. and Kang, D.S. (2001) Prevalence of Malocclusion among Latino adolescents. American Journal of Orthodontics \& Dentofacial Orthopedics, 119, 313-315. doi:10.1067/mod.2001.110985

[41] Proffit, W.R., Fields, H.W. and Moray, L.J. (1998) Prevalence of Malocclusion and orthodontic treatment need in the United States: Estimates from the NHANES III survey. International Journal of Adult Orthodontics \& Orthognathic Surgery, 13, 97. 